\title{
Scale up isolation of aaptamine for in vivo evaluation indicates its neurobiological activity is linked to the delta opioid receptor
}

\section{DOMINICAN \\ UNIVERSITY \\ of CALIFORNIA 189}

\section{Introduction}

Opioid receptors belong to the large superfamily of seven transmembrane-spanning (7TM) G protein-coupled receptors (GPCRs). As a class, GPCRs are of fundamental physiologic importance mediating the actions of the majority of known neurotransmitters and hormones. The Mu, Delta and Kappa (MOR, DOR, KOR) opioid receptors are particularly intriguing members of this receptor family as they are the targets involved in many few marine One note natural Compound $\mathbf{1}$ was the first marine natural product approved by the FDA and is used for the

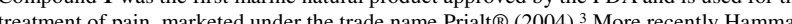
reported that aaptamine (2) is the first marine natural product to show in vivo antidepressant activity, however no mechanism of action was proposed 1,4 During a sepe collaborative screening project we profiled 96 sponge-derived extracts and discovered demethyl-aaptamine (3) and demethyl (oxy) -aaptamine (4) were selective DOR agonists as shown in Fig. 1. We speculated that the in vivo activity for $\mathbf{2}$ could thus be linked to the DOR target and to test this hypothesis we conducted the following experiments below.

CKSOGSSCSOTSYNCCRSCNOYTKRCY

ziconotide (1). Prialt @
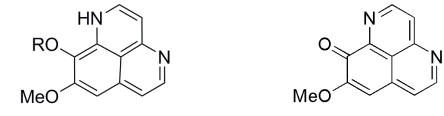

aaptamine (2), $\mathrm{R}=\mathrm{Me}$ demethyl (oxy) -aaptamine (4)

a)
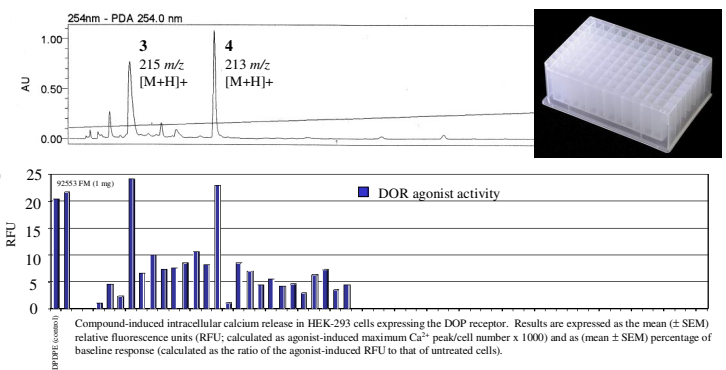

Figure 1. a) LC-MS library with annotations including $\mathrm{m} / \mathrm{z}$ ions and b) comparative DOP agonist activity of the methanol extract LC fractions of coll. no. 92553 FM.

\section{Experimental and Results}

Our first step involved obtaining a source of aaptamine (2) for in vitro and in vivo evaluation. Compounds 3-4 were obtained from the sponge Aaptos aaptos (coll. no. 92553) but were devoid of 2. LC-MS analysis of sponge coll. no. 11308 (A. aaptos) indicated $\mathrm{m} / \mathrm{z}$ using a partition scheme shown in Fig. 2. The WB extract was enriched with 2 based on LC-MS data in Fig. 3a and used to scale up it's isolation by HPLC shown in Fig. 3b. Chemical validation of pure 2 was confirmed by LC-MS and 'H NMR data in Fig. 4. This allowed us to screen 2 alongside $\mathbf{3 - 4}$ and confirm it's DOR activity in vitro. In vivo evaluation indicated $\mathbf{2}$ was an antidepressant in wild type mice in the forced swim test (Fig. 5a, black bars) while having no effect on general locomotion (Fig. 5b). We further found that the antidepressant activity was abolished in genetically modified mice where the DOR gene was knocked out (Hg. Ja, red bars, DOR KO). We also found 2 was an anxiolytic in the marble burying test (Fig. $\mathbf{~ s c ) . ~ T h e s e ~ r e s u l t s ~ i n d i c a t e ~ t h e ~ a n t i - d e p r e s s a n t ~ a c t i v i l y ~}$ previously reported for $\mathbf{2}$ is modulated by it's agonist activity of the delta opioid receptor.

\section{${ }^{1}$ Department of Natural Sciences \& Mathematics, Dominican University of California
${ }^{2}$ Department of Chemistry \& Biochemistry, University of California, Santa Cruz}

${ }^{3}$ Department of Neurology, University of California, San Francisco

†These authors contributed equally to this work

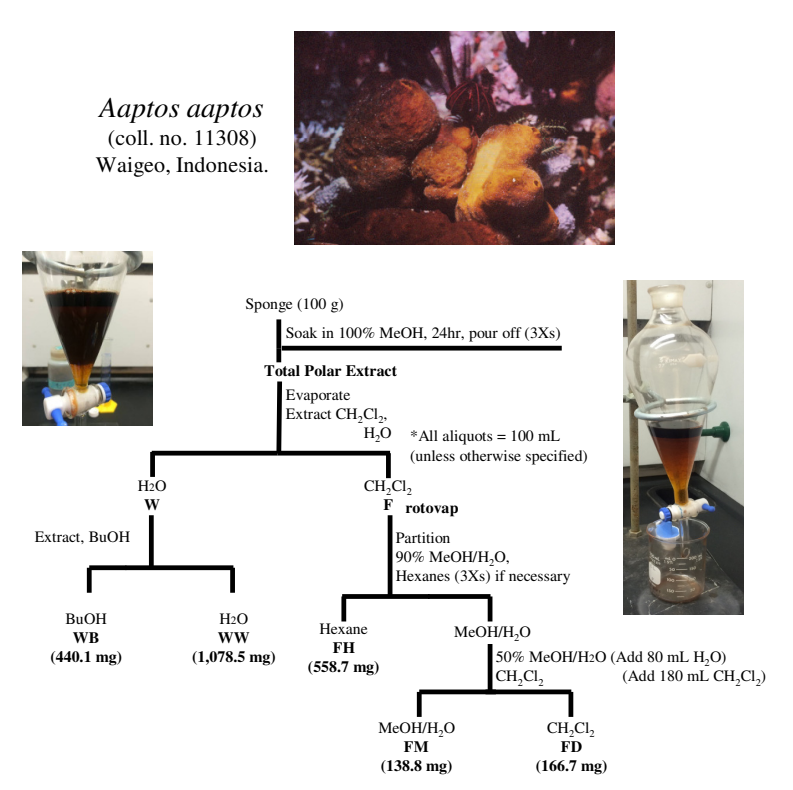

Figure 2. Extraction Scheme Flowchart of coll. no. 11308

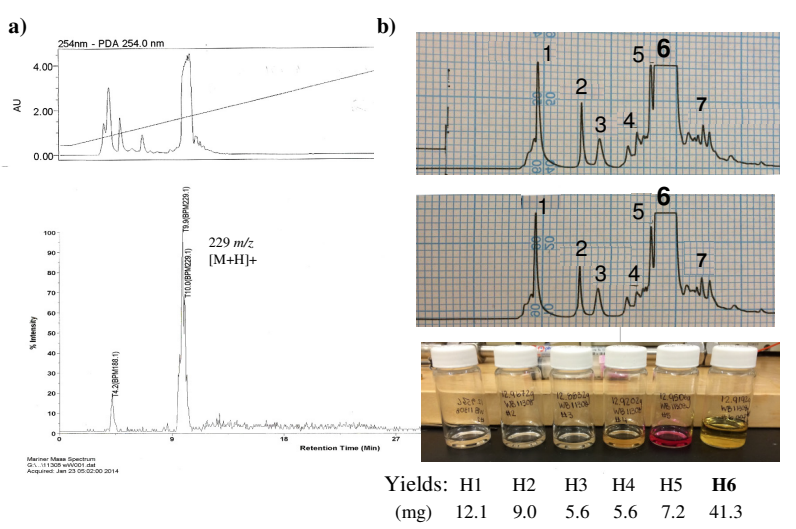

Figure 3. a) Analytical traces LC (top) MS (bottom) of coll. no. 11308 WB an b) Preparative scale up HPLC traces of coll. no. 11308 WB fractions. Gradient: $10 \% \rightarrow 100 \% \mathrm{CH}_{3} \mathrm{CN}(45 \mathrm{~min}) ;[4.0 \mathrm{mg} / 100 \mu \mathrm{l}] \times 30$ injection $\lambda_{\max }=254 \mathrm{~nm}$; sensitivity $=2.0 \mathrm{AU}$; flow $2.0 \mathrm{ml} / \mathrm{min}$.
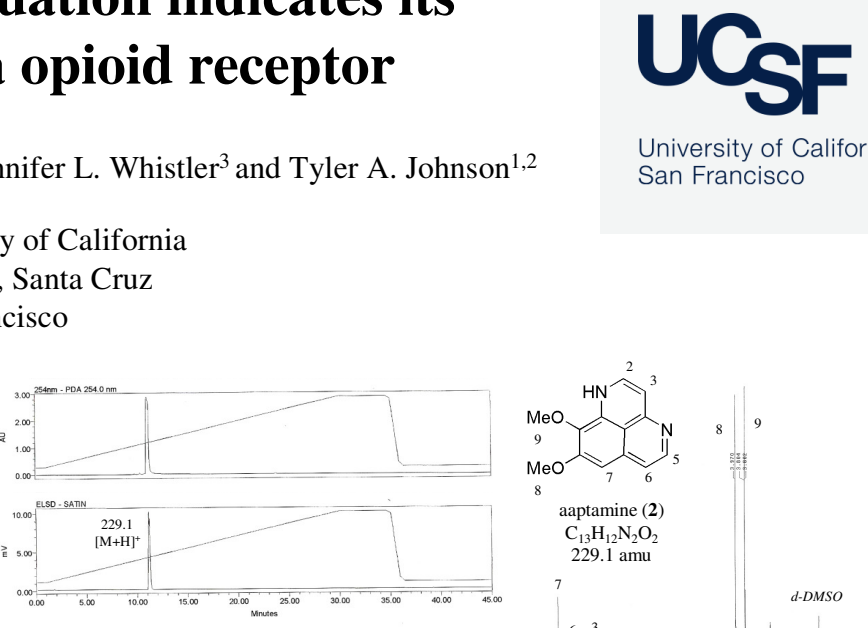

University of California San Francisco
Figure 4. Chemical validation of aptamine (2) using: a) LC-MS-ELSD analysis with annotations including $\mathrm{m} / \mathrm{z}$ ions and b) ${ }^{1} \mathrm{H}$ NMR data of 11308 WB $\mathbf{H 6}$.
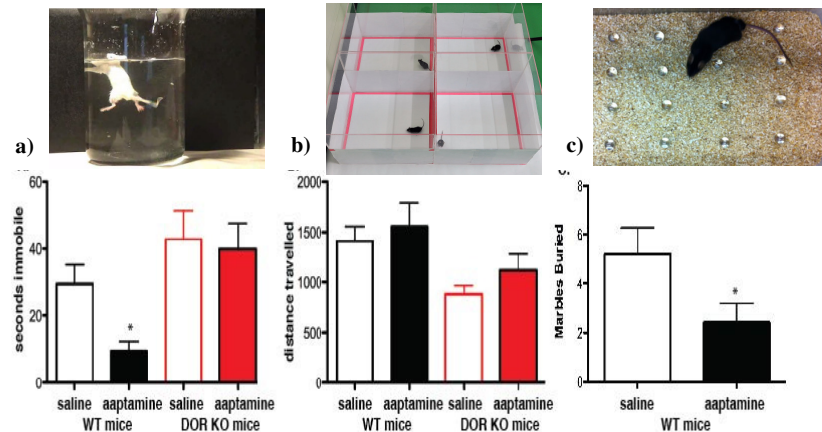

Figure 5. Mice were injected with saline or aaptamine $(2,40 \mathrm{mg} / \mathrm{kg}$, i. p) and subjected to: a forced swim test (a), a locomotor test (b) or a marble burying test (c).

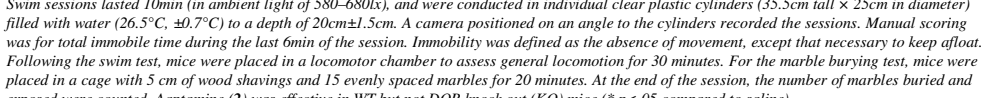

\section{Conclusions}

1) Scale up isolation of aaptamine (2) is best achieved through purification of water soluble extracts. 2) The mechanism of action for the in vivo anti-depressant-like and anxiolytic-like activity of $\mathbf{2}$ is mediated by it's activity on the delta opioid receptor (DOR).

These data suggest that 2 can represent a novel chemical scaffold for the development of new DOR ligands in neurobiological research.

\section{Acknowledgements} Financial support was provided by NIH grants RO1 CA 47135 (PC), R01 AA 020401 (JW), International Cooperative Dominice Univatit of Calforin (TJ)

\section{References}

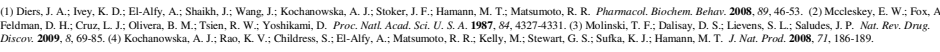

